\title{
GRIPP2 reporting checklists: tools to improve reporting of patient and public involvement in research
}

\author{
S Staniszewska, ${ }^{1}$ J Brett, ${ }^{2}$ I Simera, ${ }^{3}$ K Seers, ${ }^{1}$ C Mockford, ${ }^{4}$ S Goodlad, ${ }^{5}$ D G Altman, ${ }^{6}$ D Moher, ${ }^{7}$ \\ R Barber, ${ }^{8}$ S Denegri, ${ }^{9}$ A Entwistle, ${ }^{4}$ P Littlejohns, ${ }^{10}$ C Morris, $^{11}$ R Suleman, ${ }^{4}$ V Thomas, ${ }^{12}$ C Tysall ${ }^{4}$
}

For numbered affiliations see end of article.

Correspondence to:

S Staniszewska

sophie.staniszewska@warwick.

ac.uk

Additional material is published online only. To view please visit the journal online.

Cite this as: BMJ 2017;358:j3453 http://dx.doi.org/10.1136/bmj.j3453
GRIPP2 (short form and long form) is the first international guidance for reporting of patient and public involvement in health and social care research. This paper describes the development of the GRIPP2 reporting checklists, which aim to improve the quality, transparency, and consistency of the international patient and public involvement (PPI) evidence base, to ensure that PPI practice is based on the best evidence

ABSTRACT
BACKGROUND
While the patient and public involvement (PPI)
evidence base has expanded over the past decade,
the quality of reporting within papers is often
inconsistent, limiting our understanding of how it
works, in what context, for whom, and why.
OBJECTIVE
To develop international consensus on the key
items to report to enhance the quality, transparency,

and consistency of the PPI evidence base. To collaboratively involve patients as research partners at all stages in the development of GRIPP2.

\section{METHODS}

The EQUATOR method for developing reporting guidelines was used. The original GRIPP (Guidance for Reporting Involvement of Patients and the Public) checklist was revised, based on updated systematic review evidence. A three round Delphi survey was used to develop consensus on items to be included in the guideline. A subsequent face-to-face meeting produced agreement on items not reaching consensus during the Delphi process.

\section{RESULTS}

143 participants agreed to participate in round one, with an $86 \%(123 / 143)$ response for round two and a $78 \%(112 / 143)$ response for round three. The Delphi survey identified the need for long form (LF) and short form (SF) versions. GRIPP2-LF includes 34 items on aims, definitions, concepts and theory, methods, stages and nature of involvement, context, capture or measurement of impact, outcomes, economic assessment, and reflections and is suitable for studies where the main focus is PPI. GRIPP2SF includes five items on aims, methods, results, outcomes, and critical perspective and is suitable for studies where PPI is a secondary focus.

\section{CONCLUSIONS}

GRIPP2-LF and GRIPP2-SF represent the first international evidence based, consensus informed guidance for reporting patient and public involvement in research. Both versions of GRIPP2 aim to improve the quality, transparency, and consistency of the international PPI evidence base, to ensure PPI practice is based on the best evidence. In order to encourage its wide dissemination this article is freely accessible on The BMJ and Research Involvement and Engagement journal websites.

The EQUATOR network has developed high standard reporting guidelines such as the CONSORT (Consolidated Standards of Reporting Trials) statement and the STROBE (Strengthening the Reporting of Observational Studies in Epidemiology) statement enhancing the quality of research reporting, but no guidance has been developed specifically for the reporting of patient and public involvement (PPI). This prompted the development of the original Guidance for Reporting Involvement of Patients and the Public (GRIPP), which tackled inconsistent reporting by helping researchers, patients, carers, and the public to improve the quality, consistency, and transparency of PPI reporting, to strengthen the 
quality of the international PPI evidence base. ${ }^{1}$ While the original GRIPP checklist represented an important starting point in creating high quality PPI reporting, its development drew on systematic review evidence, without broader input from the international PPI research community. ${ }^{2-4}$ Achieving consensus is now acknowledged as a crucial step in producing a reporting guideline. ${ }^{5}$ GRIPP2 tackled this gap by developing consensus in the international PPI community.

INVOLVE defines public involvement in research as being carried out with or by members of the public rather than to, about, or for them. PPI in research can improve the relevance and overall quality of research, by ensuring that it focuses on the issues of importance to patients. ${ }^{1}$ This includes, for example, working with research funders to prioritise research; the development of more patient relevant research questions, study designs, and outcomes; offering the patient perspective as members of a project steering group; commenting on and developing research materials to improve readability; assisting with recruitment to studies; lay write up of the studies; and advocacy of study results. ${ }^{2-4}$ 6-9 In the UK, the National Institute for Health Research has provided vital strategic and infrastructure support to embed PPI across publicly funded research, creating a context where PPI is seen as a key element in research. Internationally PPI is also developing, with similar initiatives in Canada, United States, Australia, and Europe. ${ }^{10}{ }^{11}$ Networks such as the citizen and patient involvement group of Health Technology International have evolved, enabling international collaboration in relation to involvement and engagement. ${ }^{12}$

While the PPI evidence base has expanded significantly over the past decade, the reporting of PPI in papers has often been inconsistent and partial, with little information about the context, process, and impact of public involvement and with limited reporting of conceptualisation or theorisation. ${ }^{1-4}$ Inadequate reporting can create problems for systematic reviews that attempt to synthesise PPI evidence. ${ }^{2-4}$ Appraisal, interpretation, and synthesis of results are difficult, aside from the ethical imperatives of reporting research in a way that others understand and can use. ${ }^{13-16}$ Inconsistent reporting creates a fragmented evidence base making it difficult to draw together our collective understanding of what works, for whom, why, and in what context. Furthermore, researchers, patients, carers, or clinicians cannot learn from previous experience, and precious resources devoted to involving patients and the public are wasted. Omitting descriptions of PPI activities from a study can represent a form of misreporting and might misrepresent the initial intentions of a study.

This article introduces the two versions of the GRIPP2 reporting checklist: GRIPP2-LF, a longer checklist for studies where PPI forms the primary focus of a study (table 1) and GRIPP2-SF, a short checklist for studies where PPI is a secondary or tertiary focus (table 2). We also describe the development of GRIPP2 and outline how it can be used.

\section{GRIPP2 reporting checklist development methods}

The study used the EQUATOR method for developing reporting guidelines, ${ }^{5}$ which included: systematic review evidence; a three stage Delphi survey including key stakeholders in the field of PPI; and a face-toface collaborative meeting to develop consensus on items outstanding from the Delphi survey. A summary of methods is presented, with a companion paper reporting the rationale for GRIPP2 and the full methods. ${ }^{17}$ For the purposes of this paper, we have therefore reported only a summary of key steps in appendix 1 .

The systematic reviews that underpinned the original GRIPP checklist had already identified the need for the guidance. ${ }^{2-4}$ The PIRICOM systematic review, which included the conceptualisation, definition, measurement, impact, and outcomes of PPI on research, researchers, service users, participants, funders, and policy makers, was updated for GRIPP2 to ensure no additional concepts were omitted from the Delphi survey. In addition, searches were conducted to identify any other reporting guidelines for PPI.

Three rounds of the Delphi survey were conducted to gain consensus (see appendix 2). This included 143 international participants in round one, with an $86 \%(123 / 143)$ response for round two and a $78 \%(112 / 143)$ response for round three reflecting the standard number of participants used in the development of previous EQUATOR guidance. ${ }^{5}$ Participants of the Delphi survey included researchers, funders, patients, carers, editors, and individuals from international research agencies from countries including Australia, the United States, Canada, and Europe. Collectively, participants represented a wide range of expertise relevant to the development of consensus in PPI reporting.

Participants were asked to rate each item in the checklist on a scale of 1-10, with 1 considered unimportant and 10 considered very important, and medians and interquartile ranges were calculated for each item in the Delphi survey. Space next to each item was used for free text comments with suggested refinements, reiterations, and additional items. If items reached a median score of $\geq 8$ in round one and round two they were considered to have reached positive consensus and included. Items that reached a median $\leq 5$ in rounds one and two were excluded from the checklist. Items that reached a medium score of 6 or 7 in one round and a median score of $\geq 8$ in the other round were voted on again in round three. Positive consensus was gained if the items scored a median score of $\geq 8$ in two of the rounds. An important finding from the first round was that participants thought GRIPP items were most relevant when the main focus of a study was on PPI, and many felt there should be a shorter version for papers that included some element of PPI. As a result participants were asked to identify and score "core" items in round two which could be included in a shortened version of the guideline, suitable for studies that have included PPI as a secondary focus. The five core items that comprise the GRIPP2-SF all 


\begin{tabular}{|c|c|c|}
\hline Section and topic & Item & Reported on page No \\
\hline \multicolumn{3}{|l|}{ Section 1: Abstract of paper } \\
\hline 1a: Aim & Report the aim of the study & \\
\hline 1b: Methods & Describe the methods used by which patients and the public were involved & \\
\hline 1c: Results & Report the impacts and outcomes of PPI in the study & \\
\hline 1d:Conclusions & Summarise the main conclusions of the study & \\
\hline 1e: Keywords & Include PPI, "patient and public involvement," or alternative terms as keywords & \\
\hline \multicolumn{3}{|l|}{ Section 2: Background to paper } \\
\hline 2a: Definition & Report the definition of PPI used in the study and how it links to comparable studies & \\
\hline 2b: Theoretical underpinnings & Report the theoretical rationale and any theoretical influences relating to PPI in the study & \\
\hline 2c: Concepts and theory development & Report any conceptual models or influences used in the study & \\
\hline \multicolumn{3}{|l|}{ Section 3: Aims of paper } \\
\hline 3: Aim & Report the aim of the study & \\
\hline \multicolumn{3}{|l|}{ Section 4: Methods of paper } \\
\hline 4a: Design & Provide a clear description of methods by which patients and the public were involved & \\
\hline 4b: People involved & Provide a description of patients, carers, and the public involved with the PPI activity in the study & \\
\hline 4c: Stages of involvement & Report on how PPI is used at different stages of the study & \\
\hline 4d: Level or nature of involvement & Report the level or nature of PPI used at various stages of the study & \\
\hline \multicolumn{3}{|l|}{ Section 5: Capture or measurement of PPI impact } \\
\hline 5a: Qualitative evidence of impact & If applicable, report the methods used to qualitatively explore the impact of PPI in the study & \\
\hline 5b: Quantitative evidence of impact & If applicable, report the methods used to quantitatively measure or assess the impact of PPI & \\
\hline 5c: Robustness of measure & If applicable, report the rigour of the method used to capture or measure the impact of PPI & \\
\hline \multicolumn{3}{|l|}{ Section 6: Economic assessment } \\
\hline 6: Economic assessment & If applicable, report the method used for an economic assessment of PPI & \\
\hline \multicolumn{3}{|l|}{ Section 7: Study results } \\
\hline 7a: Outcomes of PPI & Report the results of PPI in the study, including both positive and negative outcomes & \\
\hline 7b: Impacts of PPI & $\begin{array}{l}\text { Report the positive and negative impacts that PPI has had on the research, the individuals involved } \\
\text { (including patients and researchers), and wider impacts }\end{array}$ & \\
\hline 7c: Context of PPI & Report the influence of any contextual factors that enabled or hindered the process or impact of PPI & \\
\hline $7 \mathrm{~d}$ : Process of PPI & Report the influence of any process factors, that enabled or hindered the impact of PPI & \\
\hline 7ei: Theory development & Report any conceptual or theoretical development in PPI that have emerged & \\
\hline 7eii: Theory development & Report testing of theoretical models, if any & \\
\hline $7 \mathrm{f}$ : Measurement & $\begin{array}{l}\text { If applicable, report all aspects of instrument development and testing (eg, validity, reliability, feasi- } \\
\text { bility, acceptability, responsiveness, interpretability, appropriateness, precision) }\end{array}$ & \\
\hline 7g: Economic assessment & Report any information on the costs or benefit of PPI & \\
\hline \multicolumn{3}{|l|}{ Section 8: Discussion and conclusions } \\
\hline 8a: Outcomes & Comment on how PPI influenced the study overall. Describe positive and negative effects & \\
\hline 8b: Impacts & $\begin{array}{l}\text { Comment on the different impacts of PPI identified in this study and how they contribute to new } \\
\text { knowledge }\end{array}$ & \\
\hline 8c: Definition & $\begin{array}{l}\text { Comment on the definition of PPI used (reported in the Background section) and whether or not you } \\
\text { would suggest any changes }\end{array}$ & \\
\hline 8d: Theoretical underpinnings & Comment on any way your study adds to the theoretical development of PPI & \\
\hline 8e: Context & Comment on how context factors influenced PPI in the study & \\
\hline 8f: Process & Comment on how process factors influenced PPI in the study & \\
\hline 8g: Measurement and capture of PPI impact & If applicable, comment on how well PPI impact was evaluated or measured in the study & \\
\hline 8h: Economic assessment & $\begin{array}{l}\text { If applicable, discuss any aspects of the economic cost or benefit of PPI, particularly any suggestions } \\
\text { for future economic modelling. }\end{array}$ & \\
\hline 8i: Reflections/critical perspective & $\begin{array}{l}\text { Comment critically on the study, reflecting on the things that went well and those that did not, so that } \\
\text { others can learn from this study }\end{array}$ & \\
\hline
\end{tabular}

$\mathrm{PPI}=$ patient and public involvement

\begin{tabular}{ll}
\hline Table 2 | GRIPP2 short form & Item \\
\hline Section and topic & Report the aim of PPI in the study \\
\hline 1: Aim & Provide a clear description of the methods used for PPI in the study \\
\hline 2: Methods & $\begin{array}{l}\text { Outcomes - Report the results of PPI in the study, including both } \\
\text { positive and negative outcomes }\end{array}$ \\
\hline 3: Study results & $\begin{array}{l}\text { Outcomes - Comment on the extent to which PPI influenced the study } \\
\text { overall. Describe positive and negative effects }\end{array}$ \\
\hline 4: Discussion and conclusions & $\begin{array}{l}\text { Comment critically on the study, reflecting on the things that went well } \\
\text { and those that did not, so others can learn from this experience }\end{array}$ \\
\hline 5: Reflections/critical perspective &
\end{tabular}

gained a median score of 9 in round two. Thus all five were included in round three and again gained median scores of 9, indicating that consensus was reached on the short form version.
Qualitative comments were analysed thematically to identify common themes, points of feedback, challenges to the items, and queries about wording. ${ }^{18}$ Qualitative comments suggested the need to reword some items 
to simplify them and ensure clarity of meaning. Two sections from the original GRIPP checklist, section 5, which focused on measurement, and section 6, which was focused on capture of impact, were combined as it was recognised that they were conceptually overlapping. The original section 8 was deleted as participants thought it duplicated existing items.

Appendices 3 and 4 report the results of the Delphi survey. Following the Delphi survey, a collaborative consensus meeting was held with 25 key experts with knowledge, experience, or both, of PPI, including patient partners and carers $(n=8)$, researchers $(n=9)$, clinicians $(n=6)$, and healthcare journal editors $(n=2)$. The aim of this meeting was to finalise consensus on the seven items on the threshold of consensus following the Delphi survey (appendix 2) and ensure clarity of the items.

Patient partners were collaboratively involved at key stages of the study. Three patient partners were recruited to the research team and were involved in refining the focus of the research questions, in development of the search strategy and interpretation of results of the systematic review, in discussions identifying the need for development of guidelines, and in selecting the items for the original GRIPP checklist. Furthermore, the patient partners assisted in developing the electronic survey for the first phase of the Delphi survey consensus process and were instrumental in assisting in recruitment to the Delphi study and in collation of comments from each Delphi survey round, and contributed to adapting items for GRIPP2. The consensus meeting involved eight patient partners in total, and the three patient partners recruited to the research team were involved in the write-up of the study and are coauthors in papers. More detailed information of their contribution to the development of GRIPP is described using GRIPP2-SF in table 3 and used to populate the BMJ PPI guidance in box 1.

\section{Scope and illustration of use}

GRIPP2-LF (table 1) and GRIPP2-SF (table 2) are the first international, evidence based, community consensus informed guidelines for the reporting of PPI in research. The checklists provide key PPI concepts

\section{Table 3 | PPI in the development of GRIPP2 using GRIPP 2-SF*}

Section and topic Item

1: Aim
Report the aim of the study

Provide a clear description of the methods used for PPI in the study

3: Results

Outcomes-Report the results of PPI in the study, including both positive and negative outcomes

\section{2: Methods}

To develop international consensus on the key items to report to enhance the quality, transparency, and consistency of the PPI evidence base. To collaboratively involve patients as research partners at all stages in the development of GRIPP2

Three patient partners were recruited to the research team to assist at all stages of the development of and consensus process for the GRIPP2 guidelines. They were involved in refining the focus of the research questions, in developing the search strategy, in interpreting results, in discussions identifying the need for development of guidelines, and in selecting the items for the original GRIPP checklist. The patient partners helped recruit participants $(n=60 / 143)$ to the Delphi survey through snowballing techniques. They helped pilot the electronic survey for the first phase of the Delphi survey consensus process and helped other patient reps with technical aspects of completing the online survey, hence improving the response rate in each round of the Delphi. They also worked with the researchers to collate comments from each Delphi survey round, to adapt items, and to feed back to the participants for the next Delphi survey round. They checked comprehension of changed items and comments from the lay perspective. The patient partners took part in the consensus workshop, alongside five other patients ( $n=8 / 25$ in total) to agree consensus on items not reaching consensus and to adapt wording where items were not clear. The patient partners contributed to edits of the paper and are coauthors.

PPI contributed to the study in several ways, including:

- Collating initial evidence

- Identifying items for the GRIPP checklist

- Considering the evidence and their wider experience-the patients highlighted the importance of including items referring to the context and processes of PPI, suggesting that this affected the impact that PPI had on research

- The patient partners, along with other patient organisations and charities, recruited nearly half of all participants for the Delphi survey

- The patient partners helped other patients with the technical aspects of completing the online survey, improving the response rate in each Delphi survey round.

- The patient partners checked the comprehension of the changed items and comments from the lay perspective between rounds and were integral to helping the researchers keep to the scheduled time of the Delphi survey

- Throughout the write-up phase for both the results paper and the methods paper the patient partners contributed to the lay sections and contributed to edits of the paper

\section{4: Discussion}

Outcomes-Comment on the extent to which PPI influenced the study overall. Describe positive and negative effects

Patient and public involvement in this study was very effective and influenced important aspects of the study, based on the impacts in section 3. This might have been related to several factors. Firstly the patient partners had received training around research methods in previous studies, and were actively involved in a patient and public involvement group attached to the University of Warwick. In addition, the researchers were experienced at involving patient partners in their research.

The right processes were in place, as the patient partners were involved from the beginning of the study allowing them to help shape the study from the start allowing them to contribute fully to the study. Having the right context, with a collaborative research team, funding to finance their time, and a supportive attitude of their involvement from EQUATOR and other collaborators, also assisted in the positive impact that PPI had on this study. Pre-existing relationships with patient partners and patients who attended the collaborative consensus event provided a vital context for embedded PPI.

However, there were limitations. The methods used to gain consensus had been developed and tested for reliability and validity by EQUATOR in the development of previous guidelines, which limited the possible input from the patient partners in identifying or developing methods to gain consensus on GRIPP2. Furthermore, the time for feedback between Delphi survey rounds was short, and organising times where both researchers and patient partners could meet was difficult. In similar future studies, scheduling of these meetings in advance of the Delphi survey might overcome this limitation.

\section{5: Reflections}

Critical perspective-Comment critically on the study, reflecting on the things that went well and those that did not, so others can learn from this experience
-The PPI in the study was embedded as far as possible into the methods for developing consensus. While not a formal part of EQUATOR methodology, the aim of active collaboration in an attempt to co-produce knowledge worked well. The key challenge was the timescales required to ensure the Delphi survey was completed with appropriate intervals. If this was repeated, these time scales would require extension. We are aware that this process might have limited the extent to which patient partners were able to identify concepts of importance that sit outside of the traditional research paradigm and so may require further development in the future. 


\section{Box 1: Patient and public involvement in GRIPP2 according to BMJ guidance}

How was the development of the research question and outcome measures informed by patients' priorities, experience, and preferences?

Patients were involved in the original systemic review that underpinned GRIPP and actively contributed to identifying the issue of inconsistent reporting, the need for guidance, and the research question.

\section{How did you involve patients in the design of this study?}

Patients were involved as research partners in all aspects of the study including identifying the original research question, identifying the need for the original systematic review, and identifying the need for consensus.

\section{Were patients involved in the recruitment to and conduct of the study?}

The patient partners, along with other patient organisations and charities, recruited nearly half of all participants for the Delphi survey. They helped pilot the electronic survey for the first phase of the Delphi survey consensus process and helped other patient reps with technical aspects of completing the online survey, hence improving the response rate in each round of the Delphi. They also worked with the researchers to collate comments from each Delphi survey round, to adapt items, and to feed back to the participants for the next Delphi survey round. They checked comprehension of changed items and comments from the lay perspective. The patient partners took part in the consensus workshop, alongside five other patients ( $n=8 / 25$ in total) to agree consensus on items not reaching consensus and to adapt wording where items were not clear. The patient partners contributed to edits of the paper and are coauthors.

How will the results be disseminated to study participants?

GRIPP2 will be disseminated to all study participants via email. The authors will disseminate via conference presentations. Funding bodies and other journal editors internationally will be encouraged to use GRIPP2.

that authors should report in papers, to enhance the overall quality and transparency of the PPI evidence base. GRIPP2-LF and GRIPP2-SF ultimately aspire to guide PPI reporting in different types of studies, from reporting on PPI in trials (GRIPP2-SF) to reporting of PPI focused studies (GRIPP2-LF). Researchers can use the reporting guideline prospectively to plan PPI in studies and retrospectively as a quality assurance step in the writing up of PPI in publications and reports. Health and social care research funders and research institutions could promote adherence to the GRIPP2 reporting checklist as a means to optimise the creation of transparent, consistent, and high quality PPI evidence. Journal editors could use GRIPP2 reporting checklists to set their reporting expectations for submitted manuscripts. Higher quality reporting will gradually lead to the development of a stronger PPI evidence base that will facilitate more effective synthesis of PPI studies.

GRIPP2 can be used in different ways within a paper. For GRIPP 2-LF the entire paper can be shaped by the guidance, with researchers selecting the items of relevance. With GRIPP2-SF researchers could present all the information in the body of the paper under the relevant reporting titles or in a separate box. Table 3 provides an illustration of GRIPP2 -SF using this study as an example. This table is an illustration of the potential of GRIPP2 reporting. It is purposefully long to demonstrate the type of information it could include. A more specific, shorter form of reporting would also be acceptable, as long as it contained the key information.

\section{Availability}

GRIPP2-SF and GRIPP2- LF are available on the EQUATOR webpage (www.equator-network.org/), or at http://www2.warwick.ac.uk/fac/med/research/ hscience/wrn/research/themea.

\section{Discussion and limitations}

GRIPP2-LF and GRIPP2-SF are the first international, evidence based, community consensus informed guidelines for the reporting of patient and public involvement in research. Although consensus was achieved in the development of GRIPP2, further refinements are expected over time as the evidence base underpinning PPI evolves, reflecting the iterative EQUATOR method of guideline development. In addition, it has not yet been possible to conduct any usability testing to understand how GRIPP2 works in practice with different types of study designs. The final consensus meeting did not include international experts because of a restricted budget, which might have limited the discussion from an international perspective. Thus, the next phase of development for GRIPP2-LF and GRIPP2-SF should include wider international application and piloting to test conceptual equivalence in different country contexts. Feedback from researchers using GRIPP2 will help refine it. We have created a comment box on the Warwick Medical School website to facilitate this http://www2.warwick. ac.uk/fac/med/research/hscience/

Guidelines such as the CONSORT statement for randomised controlled trials (RCTs) are regularly updated to reflect changes in health research more widely. ${ }^{19}$ Such evolution is particularly important for GRIPP2 because PPI is at a pre-paradigm stage in its development and recognition, reflecting Kuhn's conceptualisation of how science changes over time with significant paradigm shifts that generate new ways of thinking. ${ }^{20}$

While GRIPP2-LF and GRIPP2-SF aim to guide consistent reporting, it is not possible to be prescriptive about the exact content of each item, as the current evidence base is not advanced enough to make this possible. ${ }^{2-421}$ Authors should carefully consider the relevance of each GRIPP2 item but recognise that it is sometimes not necessary, or even possible, to include each item in a particular manuscript. With future development of the evidence base, it will be possible to refine GRIPP items, and some may become mandatory.

The success of the PPI in this study may relate to several factors. Firstly, the patient partners had received training around research methods in previous studies and were actively involved in a patient and public involvement group attached to the University of Warwick Medical School. Furthermore, the researchers were experienced at involving patient partners in their research. ${ }^{22}$ Finally, good relationships and ways of working were established, which are known as key factors for facilitating high quality PPI. ${ }^{421}$

We recognise that GRIPP2 was developed with experts familiar with PPI and that there are still significant challenges in academic culture in enacting 
the behaviour changes that public involvement requires. PPI needs to become embedded practice within research rather than an option, and both researchers and patients need to recognise their own training and development needs, drawing on the evidence base to guide effective practice.

A further limitation is that GRIPP2-LF and GRIPP2$\mathrm{SF}$ are conceptualised within the culture and language of research. Bearing in mind that the ultimate intention of high quality reporting in PPI is to develop best practice, there is a need to develop a patient or service user version of GRIPP2 to ensure comprehensibility and usefulness and to ensure that patient important concepts indicative of high quality research are included, although these are yet to be identified. This would reflect important changes in academic publishing, where patients are regularly writing and peer reviewing academic papers and require ways of understanding reporting quality. ${ }^{22}$ Used alongside other EQUATOR guidance, the intention is to guide the development of a transparent, consistent, and high quality PPI evidence base. More effective synthesis of the PPI evidence base will help to identify best practice, avoid poor practice, and contribute to research that is acceptable, relevant, appropriate, and high quality and that has the potential to generate benefit for all.

\section{AUTHOR AFFILIATIONS}

${ }^{1}$ Warwick Research in Nursing, Warwick Medical School, University of Warwick, Coventry CV4 7AL, UK

${ }^{2}$ Faculty of Health and Life Sciences, Oxford Brookes University, Oxford, UK

${ }^{3}$ Centre for Tropical Medicine and Global Health and UK EQUATOR Centre, University of Oxford, UK

${ }^{4}$ Warwick Medical School, Coventry, UK

${ }^{5}$ Coventry University, Coventry, UK

${ }^{6}$ Centre for Statistics in Medicine, University of Oxford, UK

${ }^{7}$ Centre for Journalology, Clinical Epidemiology Program, Ottawa Hospital Research Institute, Ottawa, Canada

${ }^{8} \mathrm{~S}$ chool of Health and Related Research, Section of Public Health, University of Sheffield, Sheffield, UK

${ }^{9}$ National Institute for Health Research, UCL School of Life and Medical Sciences, London, UK

${ }^{10}$ Kings College London, London, UK

${ }^{11}$ University of Exeter Medical School, Exeter, UK

${ }^{12}$ Public Involvement Programme, National Institute for Health and Care Excellence, London, UK

We thank everyone who participated in the Delphi survey and attended the consensus event. GRIPP2 was funded by the RCN Research Institute, Warwick Medical School, as part of its strategic focus on patient and public involvement. We thank Sally Crowe who facilitated the consensus workshop. SS is part funded by the National Institute for Health Research (NIHR) Collaboration for Leadership in Applied Health Research and Care West Midlands. PL is supported by the National Institute for Health Research (NIHR) Collaboration for Leadership in Applied Health Research and Care South London at King's College Hospital NHS Foundation Trust. This paper presents independent research and the views expressed are those of the author(s) and not necessarily those of the NHS, the NIHR or the Department of Health.

Contributors: SS, JB, IS, KS, C Mockford, SG, C Morris, SD, RB, PL, VT, RS, AE, and CT made substantial contributions to conception and design. JB, SS, IS, DGA, and DM made substantial contributions to developing the protocol. SS, JB, and SG made substantial contributions to the acquisition of data, analysis, and interpretation of data. All authors have been involved in drafting the manuscrip or revising it critically for important intellectual content; given final approval of the version to be published.
Funding: The development of GRIPP2 was recognised as strategically important and funded by Royal College of Nursing Research Institute, Warwick Medical School, University of Warwick.

Ethical approval: Ethical approval for the study was secured by the Centre for Educational and Industry/Development, Appraisal and Research (CEI) at the University of Warwick, which gained generic approval from the University Ethics Committee for all of its online surveys. The University Ethics Committee reviewed the rigorous survey procedures CEI had in place and granted generic ethical approval for its robust process and procedures. The GRIPP2 Delphi survey was assessed by CEI as being covered by the generic approval. Complete details of ethical procedure and methods are reported in the companion paper. ${ }^{17}$

Competing interests: SS is co-editor in chief of Research Involvement and Engagement, and so Richard Stephens handled this manuscript for Research Involvement and Engagement. No other author has declared a competing interest.

This is an Open Access article distributed in accordance with the terms of the Creative Commons Attribution (CC BY 4.0) license, which permits others to distribute, remix, adapt and build upon this work, for commercial use, provided the original work is properly cited. See: http://creativecommons.org/licenses/by/4.0/.

1 Staniszewska S, Brett J, Mockford C, Barber R. The GRIPP checklist strengthening the quality of patient and public involvement reporting in research. Int I Technol Assess Health Care 2011:27:391-9. doi:10.1017/S0266462311000481

2 Brett J, Staniszewska S, Mockford C. Mapping the impact of patient and public involvement on health and social care research: a systematic review. Health Expect 2014;17:637-50. doi:10.1111/ j.1369-7625.2012.00795.x

3 Mockford C, Staniszewska S, Griffiths F, Herron-Marx S. The impact of patient and public involvement on UK NHS health care: a systematic review. Int J Qual Health Care 2012;24:28-38. doi:10.1093/intqhc/ mzr066.

4 Brett J, Staniszewska S, Mockford C. A systematic review of the impact of patient and public involvement on service users, researchers and communities. Patient 2014;7:387-95. doi:10.1007/s40271-0140065-0.

5 Moher D, Schulz KF, Simera I, Altman DG. Guidance for developers of health research reporting guidelines. PLoS Med 2010;7:e1000217. doi:10.1371/journal.pmed.1000217

6 Domecq JP, Prutsky G, Elraiyah T. Patient engagement in research: a systematic review. BMC Health Serv Res 2014;14:89. doi:10.1186/1472-6963-14-89

7 Shippee ND, Domecq Garces JP, Prutsky Lopez GJ. Patient and service user engagement in research: a systematic review and synthesised framework. Health Expect 2013;18:1151-66.

8 Gagnon MP, Desmartis M, Lepage-Savary D. Introducing patients' and the public's perspectives to health technology assessment: A systematic review of international experiences. Int J Technol Assess Health Care 2011;27:31-42. doi:10.1017/ S0266462310001315

9 Staley K. Exploring impact: public involvement in NHS, public health and social care research. INVOLVE: Eastleigh. 2009 http://www.invo. org.uk/posttypepublication/exploring-impact-public-involvement-innhs-public-health-and-social-care-research/

10 National Institute for Health. Going the extra mile: improving the nation's health and wellbeing through public involvement in research. 2015. https://www.nihr.ac.uk/about-us/documents/ Extra\%20Mile2.pdf

11 Tarrow S. Power in movement: Social movements, collective action and politics.Cambridge University Press, 1994.

12 Health Technology Assessment International Patient and Citizen Involvement subgroup http://www.htai.org/interest-groups/patientand-citizen-involvement.html.

13 Moher D. Reporting research results: a moral obligation for all researchers. Can J Anaesth 2007;54:331-5. doi:10.1007/BF03022653

14 Moher D. Guidelines for reporting health care research: advancing the clarity and transparency of scientific reporting. Can I Anaesth 2009;56:96-101. doi:10.1007/s12630-008-9027-0

15 Little J, Higgins J, Ioannidis JPASTROBE Extension to Generic Association Studies. STREGA (STrengthening the reporting of generic association studies). PLoS Med 2009;6:e22. doi:10.1371/journal. pmed. 1000022

16 Bossuyt PM, Reitsma JB, Bruns DEStandards for Reporting of Diagnostic Accuracy. Towards complete and accurate reporting of studies of diagnostic accuracy: The STARD Initiative. Ann Intern Med 2003;138:40-4. doi:10.7326/0003-4819-138-1200301070-00010

17 Brett J, Staniszewska S, Simera I. Reaching consensus on reporting patient and public involvement (PPI): lessons learned 
from the development of reporting guidelines. BMJ Open (forthcoming).

18 Braun V, Clarke V. Using thematic analysis in psychology. Qual Res Psychol 2006;3:77-101doi:10.1191/ 1478088706qp0630a.

19 Moher D, Hopewell S, Schulz KF. CONSORT 2010 explanation and elaboration: updated guidelines for reporting parallel group randomised trials. BMJ 2010;340:c869. doi:10.1136/ bmj.c869

20 Kuhn TS. The structure of scientific Revolutions. 50th anniversary. lan Hacking (Introd.).4th ed. University of Chicago Press, 2012: 264doi:10.7208/chicago/9780226458144.001. 0001.
21 Wilson P, Mathie E, Keenan J. ReseArch with Patient and Public involvement: a RealisT evaluation-the RAPPORT study. Health Services and Delivery Research 2015;3. doi:10.3310/hsdr03380.

22 Stephens R, Staniszewska S. One small step.... Research Involvement and Engagement 2015:1. doi:10.1186/s40900-015-0005-8.

Appendix 1: Flowchart of the Delphi survey Appendix 2: Consensus meeting outcomes Appendix 3: Results of the Delphi survey for GRIPP2 long form

Appendix 4: Results of the Delphi survey for GRIPP2 short form 